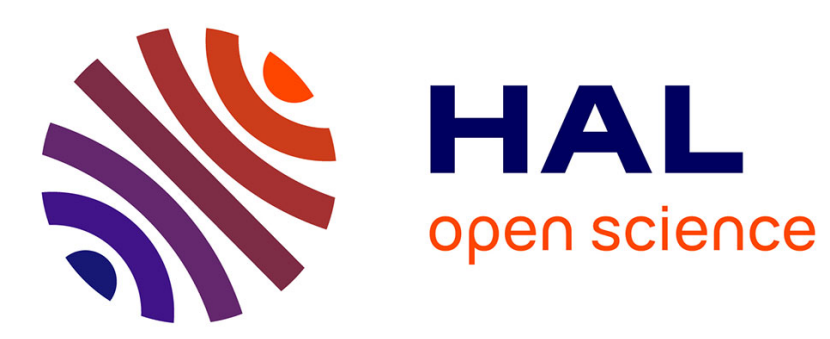

\title{
Contributions historiennes au renouveau de la sociologie de l'État. Regards croisés franco-américains
}

\author{
Sarah Gensburger
}

\section{To cite this version:}

Sarah Gensburger. Contributions historiennes au renouveau de la sociologie de l'État. Regards croisés franco-américains. Revue française de sociologie, 2011, 52 (3), pp.579-602. halshs-00828009

\section{HAL Id: halshs-00828009 \\ https://shs.hal.science/halshs-00828009}

Submitted on 29 Nov 2013

HAL is a multi-disciplinary open access archive for the deposit and dissemination of scientific research documents, whether they are published or not. The documents may come from teaching and research institutions in France or abroad, or from public or private research centers.
L'archive ouverte pluridisciplinaire HAL, est destinée au dépôt et à la diffusion de documents scientifiques de niveau recherche, publiés ou non, émanant des établissements d'enseignement et de recherche français ou étrangers, des laboratoires publics ou privés. 
"Contributions historiennes au renouveau de la sociologie de l'Etat. Regards croisés franco-américains ", Revue française de sociologie, rubrique Note critique, printemps 2011, p. 579-602.

\section{Contributions historiennes au renouveau de la sociologie de l'Etat. Regards croisés franco-américains.}

Sarah Gensburger

CNRS - Institut de Sciences Sociales du Politique

La sociologie de l'Etat connaît aujourd'hui un profond renouvellement. Ce dernier prend, pour une large part, appui sur les conclusions des travaux historiens qui se multiplient depuis une dizaine d'années. Ceux-ci invitent en effet à rompre avec une approche unidimensionnelle de l'Etat d'ordinaire exprimée en termes de «plus » ou «moins ». Tandis que les historiens français «découvrent » la présence de la société au cœur même de cet Etat "fort» qu'est censée incarner la France jacobine, leurs collègues américains «révèlent» l'interventionnisme social d'un Etat supposé « faible »"

Dans l'Hexagone comme Outre-Atlantique, cet intérêt des historiens pour l'Etat est relativement récent. En 1989, encore, Pierre Rosanvallon déplorait le fait que l'Etat demeurait un «non-objet historique» (Rosanvallon, 1990 [1989], p. 9) tandis que, trois ans plus tôt, pour William Leuchtenburg, alors président de l'Organization of American Historians, l'Etat demeurait un domaine à conquérir, une «new frontier », pour sa discipline (Leuchtenburg, 1986, p. 589). Le " retour aux acteurs » a, depuis, conduit les historiens à rencontrer l'Etat (Revel, 1995 ; Guery, 1997 ; Boucheron, 1998 ; Chatriot, 2006). Aux Etats-Unis, « social and cultural historians followed their stories inside City Hall [and] state houses » (Balogh, 2003, p. 458$)^{2}$. En France, et pour ne prendre que l'exemple de l'histoire moderne, au demeurant pionnière sur cette thématique, la focale s'est, de même, peu à peu déplacée : de l'attention aux facteurs de la formation des Etats (Tilly, 1975 et 1990) à celle aux simples « sujets » du roi (Te Brake, 1998 ; Gorski, 2001).

$\mathrm{Si}$, dans les deux pays, ces travaux aboutissent à des reformulations théoriques majeures, ils connaissent cependant des institutionnalisations différenciées: au sein de la discipline historique, d'une part, dans les relations qu'ils entretiennent avec la sociologie et la science politiques, de l'autre. Dans l'Hexagone, l'intérêt des historiens du contemporain pour cette thématique a été encouragé par la création de comités d'histoire dont la fin des années 80 a vu la multiplication, à l'image du Comité d'histoire économique et financière instauré en 1986 par le Ministère de l'Economie, des Finances et du Budget (Margairaz, 1991). A la même époque, des financements publics permettent la conduite d'un important programme européen sur la " Genèse de l'Etat moderne », consacrant le rôle central de l'histoire moderne dans le développement de recherches historiennes sur 1'Etat (Genet, 1997 ; Schaub, 1996 ; Descimon et Guéry, 1989). Peut-être du fait de cette césure entre historiens aux périodes de spécialisation différentes et malgré un certain nombre de réflexions historiographiques sur ce qui prend peu à peu la forme d'une « histoire de l'Etat »(Chatriot, 2006 ; Fridenson, 2000), cette dernière n'a toutefois pas connu de véritable institutionnalisation. Cet état de fait va de pair avec la faiblesse du dialogue de ces travaux historiens avec ceux produits par la sociologie et la science politiques, à l'image du peu de place que les premiers donnent aux

\footnotetext{
${ }^{1}$ Le choix de restreindre ce texte aux travaux américains et français découle donc avant tout de la nature archétypale de ces deux cas. Une évolution comparable se retrouve cependant ailleurs. En Allemagne, par exemple, l'Etat a cessé d'être un des facteurs pris en compte dans l'histoire sociale pour devenir l'objet même de l'investigation (Gosewinkel, 2006).

${ }^{2}$ Ce premier groupe de travaux américains porte notamment sur le genre (Gordon, 1994), la conquête de l'Ouest (White, 1991) ou encore les questions environnementales (Hays, 1987).
} 
recherches qui s'inscrivent dans la mouvance de la socio-histoire (Quennouëlle, 2002) ${ }^{3}$. A cet égard, les publications les plus récentes marquent une rupture. Ce n'est pas, alors, la sociologie de l'Etat proprement dite mais l'analyse des politiques publiques qui est mobilisée. Tandis que cette dernière cherche, désormais, elle-même à opérer un retour au concept d'Etat, elle est vue ici, et donc de manière paradoxale, comme un moyen de renouveler l'approche historienne en la matière (Capuano, 2009 ; Delalande, 2009).

Dans le cas américain, le chemin est inversé ${ }^{4}$. Un intérêt historien pour l'Etat se fait d'abord jour dans les années 70, pour partie du fait de la pénurie d'emplois académiques offerts aux historiens. Ce Public History Movement aboutit alors à la création d'une association professionnelle et d'une revue, The Public Historian. Ce n'est cependant qu'à la fin de la décennie que cette évolution rejoint véritablement la recherche universitaire. En 1987, une nouvelle revue, cette fois-ci strictement académique, est créée. Si The Journal of Policy History ne traite pas uniquement de l'Etat, la question de la nature, du fonctionnement et des recompositions de celui-ci y tient une place croissante, aujourd'hui centrale. Cette institutionnalisation réussie d'un sous-champ tient d'abord à l'importance du dialogue de ces historiens avec l'institutionnalisme historique qui a connu un développement exponentiel au sein de la science politique américaine ${ }^{5}$. Comme le résume J. Zelizer, historien et directeur du Journal of Policy History: "Given the state of politics within the history profession, some of the most prominent political historians by the 1990s tended to be political scientists » (Zelizer, 2004, p. 128). Aujourd'hui les échanges demeurent constants entre l'institutionnalisme historique, et plus largement l'American Political Development, et les travaux historiens qui traitent de l'Etat ${ }^{6}$. La question de la continuité et du changement est au cœur de ce dialogue. L'enjeu pour les historiens est ainsi de déterminer : d'un côté, s'il est pertinent de parler de state building, c'est-à-dire d'un début et d'une fi de l'autre, si l'Etat - en l'espèce américain - a le même sens, le même but et le même pouvoir selon les époques. Cette interrogation se retrouve dans l'Hexagone - et donc dans un tout autre paysage académique : «Tout se passe en effet comme si l'on considérait que l'Etat n'avait pas véritablement d'histoire, son développement n'étant que la pure reproduction, sans cesse agrandie, d'une figure qui aurait été formée à l'origine » (Rosanvallon, 1990, p. 10).

Ainsi, malgré l'éclatement des travaux historiens qui traitent de l'Etat en France et la reprise par les historiens américains de nombre des questionnements de l'American Political Development, des axes transversaux apparaissent. Ils constituent autant de perspectives à partir desquelles enrichir la reformulation en cours de la sociologie de l'Etat. Ceux-ci relèvent d'approches de l'Etat, respectivement organisationnelle, relationnelle et cognitive.

\section{La remise en cause d'une approche unidimensionnelle de l'Etat}

«Les Etats ne sont pas tant plus forts ou plus faibles les uns des autres que qualitativement différents » (Baldwin, 2005, p. 13). Cette citation résume parfaitement la conclusion principale à laquelle les travaux historiens sur l'Etat ont abouti ces dernières années. Ils sont ainsi le plus souvent comparatistes. Dans Birth of the Leviathan, T. Ertman reprend, par

\footnotetext{
${ }^{3}$ Comme le montrent, par exemple et de manière exemplaire, la bibliographie et la table des matières de Baruch et Duclert (2000).

${ }^{4}$ Pour une histoire détaillée du développement de ce sous-champ (Zelizer, 2000 ; Graham, 1993).

${ }^{5}$ Sur ce point voir la discussion organisée à l'occasion des cinquante ans de la parution de Building a New American State (Balogh, 2003 ; Zelizer, 2003).

${ }^{6}$ Pour un débat sur ce point (Zelizer, 2005 ; Pierson, 2005).
} 
exemple, la thèse classique du rôle de la guerre dans la naissance de l'Etat pour s'interroger plus spécifiquement sur les causes de la différenciation entre les régimes dotés d'administrations bureaucratiques et ceux structurés autour d'une dynamique principale de patrimonialisation (1997). Il conclut sur la nécessité de dépasser l'opposition entre Etat absolutiste et Etat constitutionnaliste pour proposer une nouvelle typologie qui croise deux axes : absolutisme/constitutionnalisme (regime structure) et patrimonialisme/bureaucratie (administrative infrastructure). Cette nouvelle catégorisation conduit à un renversement de perspective. L'Etat français habituellement considéré comme un bureaucratique apparaît à structure patrimoniale, au motif que la dépatrimonialisation y a été particulièrement tardive. A l'inverse, l'Etat anglais, d'ordinaire caractérisé par son peu d'interventionnisme, devient un Etat doté d'une bureaucratie protomoderne (Mann, 2000, p. 333-334). Les travaux comparatifs dirigés par J. Brewer et E. Hellmuth mettent, de même, en évidence les limites théoriques de l'opposition traditionnelle entre les Etats respectivement prussien (bureaucratique, absolutiste, militaire) et britannique (constitutionnaliste, parlementaire, commercial) (1999). Mais, même lorsque les recherches historiennes sur l'Etat sont d'ordre monographiques, elles ont pour horizon implicite la comparaison entre des Etats américain ou anglais, tous deux traditionnellement caractérisés par leur faiblesse au regard d'une "société civile » qui tient le premier rôle, et un Etat français, vu comme le modèle du "véritable Etat », doté d'une administration forte imperméable aux corps intermédiaires. A cet égard, la remise en cause simultanée de ces deux visions archétypales marque un tournant.

\section{La « découverte » de l'interventionnisme de l'Etat américain}

Dans cette évolution, l'historiographie américaine joue un rôle moteur. La vulgate historienne a longtemps considéré que l'Etat fédéral américain était traditionnellement inexistant et que le principe du laissez-faire caractérisait son action. Seul le tournant de la Progressive Era du début du $20^{\text {ème }}$ siècle et surtout la mise en place du New Deal dans l'entre-deux guerres auraient donné naissance à un véritable Etat qui, malgré des signes de puissance au niveau international, aurait continué à être en retrait. Bien entendu les travaux de Stephen Skowronek (1982) ont marqué une première remise en cause de cette lecture. Mais ils se limitent au $19^{\text {ème }}$ siècle et, tout en affirmant l'existence et l'autonomie de l'Etat, le décrivent comme structuré par les partis et les tribunaux et donc dans un état d'incomplétude par rapport aux Etats européens, français ou allemand. Ainsi les recherches historiennes ont depuis mis en évidence l'existence d'un Etat administré et centralisé et ce bien avant la fin du $19^{\text {ème }}$ siècle. "The American state is and always has been more powerful, capacious, tenacious, interventionist, and redistributive than was recognized in earlier accounts of U.S. history" (Novak, 2008, p. 758). L'histoire du droit a été pionnière dans cette révision historiographique. L'ouvrage de W. Novak compte parmi ceux qui ont ouvert la voie (1996) ${ }^{7}$. Il s'appuie sur un corpus juridique, qui avait jusqu'ici été presque totalement négligé par les historiens, pour mettre en évidence l'interventionnisme de l'Etat dans les domaines de l'économie, de l'urbanisme, des transports, des mœurs, de l'hygiène ou encore de la santé publique. Il forge alors le concept de "well-regulated society » pour décrire la manière dont aux Etats-Unis les intérêts et les droits des individus sont effectivement encadrés par l'Etat. Novatrice, cette recherche laisse cependant ouverte tant la question des effets de cette réglementation abondante que celle de la construction par l'Etat de non-droits et de discriminations.

\footnotetext{
${ }^{7}$ Plus récemment, J.L. Mashaw a mis en évidence l'existence, dès la création des Etats-Unis, d'un véritable droit administratif qui témoigne de la présence d'une administration centralisée (2005).
} 
Des études thématiques qui mêlent sources législatives et archives de leur mise œuvre ont depuis comblé cette lacune. L'Etat apparaît alors comme ayant fortement encadré, permis et organisé le développement économique au pays du « laissez-faire ». L'étude de la création du système postal par R. John (1995) fait à cet égard figure de classique. L'auteur y montre comment le développement d'un réseau postal est l'œuvre du gouvernement fédéral et y insiste sur le rôle central que celui-ci a joué dans la naissance d'une économie nationale, en dessinant, par exemple, les voies de communications ou le rythme de la vie collective. L'ouvrage de R. F. Bensel décrit de même, et de manière systématique, comment l'industrialisation des Etats-Unis a été encadrée, portée et facilitée par l'Etat fédéral (1991 et $2000)^{8}$. Un second grand domaine d'étude concerne, lui, la délimitation de l'espace national et de ses frontières ${ }^{9}$. L'ouvrage de R. White montre comment, loin d'être un lieu de non-droit, l'Ouest américain fut une création continue du gouvernement fédéral (1991). En retour, son développement a structuré la mise en place d'une véritable bureaucratie, importante en nombre et répartie sur l'ensemble du territoire à tel point que, symbole de l'individualisme américain, l'Ouest apparaît comme le laboratoire du «big government ». Parmi les politiques mises en œuvre par l'administration figure, également, celles qui visent à la catégorisation des citoyens et des non-citoyens. La mise en place de l'esclavage puis de la ségrégation raciale constitue ainsi une illustration particulièrement saisissante de l'existence d'un Etat interventionniste aux Etats-Unis (Fehrenbacher, 2001 ; King, 1995 ; Ngai, 2005). Lié à cette question, le "police power », qui va jusqu'au contrôle des mœurs et des comportements intimes, constitue enfin un important champ d'investigation. Ces tentatives de contrôle étatique sur le privé, jusqu'à l'intime, manifestent ainsi avec d'autant plus de force l'existence d'un Etat américain bien avant le début du $20^{\text {ème }}$ siècle comme l'ampleur de son interventionnisme comparé à celui de ses équivalents européens davantage en retrait sur ces questions (Stearns, 1999 ; Barringer Gordon, 2002 ; Tomlins, 2008).

\section{L'entre-deux britannique}

Le changement de paradigme se retrouve à l'identique dans l'étude du cas britannique. Les travaux de M. Daunton (2001 et 2002) sur la mise en place de la taxation en Grande-Bretagne ont ainsi montré comment les institutions britanniques - et notamment la place qui tient l'articulation entre gouvernement central et pouvoirs locaux - ont permis le développement, au $19^{\text {ème }}$ siècle, d'un système fiscal qui a rendu possible la collecte d'une part du revenu national bien supérieure aux prélèvements alors effectués par l'Etat français mais aussi la contraction d'emprunts d'Etat par un nombre de citoyens largement supérieur ou encore la mise en place d'impôts directs plus tôt et plus largement ${ }^{10}$. Dès lors que l'on considère que le prélèvement fiscal et le consentement à l'impôt comptent parmi les principaux critères d'existence d'un Etat, il apparaît qu'une plus faible institutionnalisation de l'Etat britannique va de pair avec une large emprise sur la société ${ }^{11}$. L'étude de ce que l'on appellerait aujourd'hui des politiques de santé publique aboutit aux mêmes conclusions. La mise en place, à l'époque des grandes épidémies, par l'Allemagne et la France de contrôles et de restrictions aux frontières a jusqu'ici été interprétée comme le signe de la puissance de ces Etats, l'absence de mesures comparables pour le cas britannique constituant un symptôme de la faiblesse de cet Etat. Les travaux de P. Baldwin (1999) renversent la perspective pour

\footnotetext{
${ }^{8}$ Voir également (John, 2006).

${ }^{9}$ Dans cette catégorie figurent les travaux sur la Civil War dont le rôle fut fondamental dans le développement de l'Etat américain (Wilson, 2006).

${ }^{10}$ Pour la période précédente voir (Braddick, 1996).

${ }^{11}$ Voir également (Stasavage, 2003).
} 
montrer que cet interventionnisme à la marge du territoire n'a fait que compenser l'impuissance des administrations allemande et française à mettre en place les actions nécessaires à la prise en charge de l'épidémie à l'intérieur du pays. A l'inverse, forts de leur expérience en matière d'hygiène publique et de lutte contre l'insalubrité, les Britanniques ont disposé d'un ensemble de services dédiés, dépendant de l'Etat central mais organisés dans un rapport articulé avec les pouvoirs locaux et les villes, ce qui leur a permis de laisser franchir leur frontière, y compris aux malades. Vu sous cet angle, l'Etat britannique apparaît comme clairement interventionniste: "A strong state, one might be tempted to conclude, is not seen, while a weak one flails about noisily" (Baldwin, 2005, p. 24). Ce type de travaux a donné naissance à une nouvelle hypothèse : "still interventions run deep », en d'autres termes, l'invisibilité de l'Etat pourrait être un signe de son omniprésence.

Plus largement, ces deux travaux d'envergure invitent à substituer une approche qualitative et pluridimensionnelle de l'Etat à l'approche quantitative longtemps dominante. Elles convergent sur ce point avec la réflexion de C. Laborde qui porte également sur le cas anglais mais relève, elle, de l'histoire des idées politiques. C. Laborde s'intéresse à l'utilisation du terme même d' "Etat» dans le débat politique anglais (2000). A partir de cette étude de cas, elle renvoie dos à dos les tenants de l'idée que l'Angleterre serait non seulement sans Etat mais qu'elle ne connaîtrait pas jusqu'au concept même d'Etat (Nettl, 1968 ; Badie et Birnbaum, 1979; Dyson, 1980) et leurs contestataires qui, preuves à l'appui, montrent que l'Angleterre a connu historiquement un tel concept - entendu comme Etat fort bureaucratique - dans son débat politique (Meadowcroft, 1995). Ces travaux montrent en effet que le concept d'Etat n'est pas plus présent en France qu'en Angleterre, ni l'inverse, mais que le même mot désigne des réalités différentes que l'étude des représentations permet de saisir. D'où, une fois de plus, une invitation à emprunter de nouvelles voies pour penser l'Etat: "La recherche comparative sur le concept d'Etat serait affinée, dès lors, si au lieu de distinguer entre traditions "avec Etat» et traditions «sans Etat», on s'attachait à analyser les différents degrés et types de contestabilité du concept dans des traditions différentes. Et qui sait si, dans cette optique, les étrangers ne nous apparaîtront, non plus comme désespérément dépourvus, mais plutôt comme différemment dotés » $(2000$, p. 82$)$.

\section{Retour critique sur la caractérisation de l'Etat français comme modèle}

On l'aura compris, dans ces travaux, l'Etat français constitue le canon du véritable Etat. Il est donc frappant de constater que cette remise en cause de la caractérisation des Etats américain et britannique comme des Etats faibles, en retrait voire inexistants, est exactement concomitante d'un retour critique sur l'interventionnisme et la force supposés de cet Etat français. Dans son ouvrage Origins of the French Welfare State, P. V. Dutton met, par exemple, à mal la vision d'un Etat centralisé et jacobin où l'intérêt général et le traitement universaliste prévaudraient. A travers l'étude de la mise en place des allocations familiales et de l'assurance sociale, il montre, au contraire, que l'Etat social français s'est construit à travers une négociation permanente avec un grand nombre d'acteurs collectifs, au premier rang desquels les sociétés d'aide mutuelle et les associations familiales (2002 et 2007).

Au-delà de cet ouvrage majeur, depuis quelques années, les travaux se multiplient pour nuancer l'idée même de « différenciation », centrale pour la sociologie de l'Etat, et finalement inviter au dépassement de l'opposition traditionnelle entre « jacobinisme » et « corporatisme » (Lemercier, 2005a). Le rôle de relais mais aussi d'expertise ou encore d'informateurs joués par plusieurs organisations ou groupements privés dans la conduite et la détermination de l'action de l'Etat au $19^{\text {ème }}$ siècle a ainsi été mis en évidence (Kaplan et Minard, 2004). De même, la notion d' « impératif de gouvernabilité » a été forgée pour expliquer le statut donné, 
au cœur même de l'interventionnisme étatique et dès l'origine, à certaines organisations détentrices d'un savoir et d'une compétence dont l'administration est le plus souvent dépourvue (Rosanvallon, 2004) ${ }^{12}$. Rejoignant de nombreux travaux de micro-histoire, ces travaux insistent tous sur la perméabilité qui existe entre Etat et notables. Situées à une échelle différente, mais portant également sur le XIX siècle ${ }^{13}$, les recherches de M. Gribaudi sur l'administration arrivent à un constat identique. Dans un article récent, il étudie, par exemple, les 534 employés et fonctionnaires qui ont travaillé dans les bureaux chargés de la tutelle des organismes de la Santé et de l'Hygiène publiques au ministère de l'Intérieur et à celui de l'Agriculture et du Commerce entre 1803 et 1910. Il montre alors que "loin d'apparaître comme un organisme bien structuré et délimité par ses fonctions, l'administration se révèle donc plutôt comme une ruche bourdonnante et totalement intégrée à l'intérieur d'une société qui se transforme sous le tiraillement de forces contradictoires. [...]Le fonctionnement administratif lui-même ne peut sortir indemne de ces transformations; il n'est pas non plus autonome par rapport aux tensions, tant profondes que souvent invisibles, produites par les différentes appartenances sociales originaires des employés, par les relations qu'ils nouent aussi à travers l'administration, par la configuration complexe et variable dans laquelle ils sont inscrits au fil de leur carrière » (Gribaudi, 2009, pp. 37-38).

\section{Vers une nouvelle théorisation de l'Etat}

Ce cheminement croisé des deux historiographies nationales donne aujourd'hui naissance à deux interrogations convergentes. Pour ce qui concerne l'Etat américain, la question n'est plus « why is it so weak in comparison to some European equivalents, but why is so much of it arranged outside the state?» (Baldwin, 2005, p. 15). Pour ce qui concerne l'Etat français, le «problème théorique » devient, réciproquement, celui de «l'organisation des rapports entre Etat et société civile » (Lemercier, 2005a, p. 169). Si cette reformulation des questionnements entraîne une remise en cause de plusieurs des concepts traditionnels de la sociologie de l'Etat, au premier rang desquels celui de différenciation, elle ne rompt pas, loin s'en faut, avec la théorie weberienne de l'Etat. Elle ouvre, au contraire, de nouvelles pistes pour comprendre comment s'exercent, effectivement, la contrainte et le pouvoir étatiques, fût-ce à travers une hybridation entre public et privé (Baldwin, 2003). Toutefois cette révision historienne n'a pas, pour l'heure, abouti de réelles propositions théoriques alternatives. Cette absence ne s'explique que très partiellement par le goût pour le particulier et la contextualisation, toujours singulière, qui caractérise la discipline historique. Les politistes américains qui dialoguent avec les historiens déplorent également ce manque d'explication intégrée même s'ils tentent, avec peut-être davantage de systématisme, de faire émerger de nouvelles grilles d'analyse (Hacker, 2005 ; King et Lieberman, 2009). Dans ce qui suit, il sera donc question d'approches historiennes de l'Etat plutôt que de théories ou de modèles et même si certaines d'entre elles peuvent faire l'objet d'explicitation formelle. Ces trois approches sont distinguées ici sur le mode idéal-typique et entretiennent, bien sûr, des liens étroits.

\section{L'Etat comme organisation: de la nécessité des jeux d'échelle}

\footnotetext{
${ }^{12}$ Dans la même perspective, et dans le domaine cette fois-ci de la science politique, en 1980, commentant la sortie alors récente de l'ouvrage devenu classique de P. Birnbaum et B. Badie, G. Lavau considérait déjà que «plus on voit fonctionner le couple conceptuel « société civile» - «Etat», plus grandit la perplexité. D'abord parce qu'il n'est pas sûr que ce couple conserve beaucoup de sens lorsqu'on le détache des configurations théoriques d'ensemble (chez Hegel, chez Marx, chez Gramsci) à l'intérieur desquelles les éléments de ce couple faisaient sens $»(1980$, p. 410)

${ }^{13}$ Egalement (Gribaudi, 1999 et 1998). Dans ce domaine, voir bien sûr la recherche pionnière (C. Charle, 1987).
} 
La première est la plus explicite et peut-être la plus révisionniste en ce qu'elle rompt avec la conception de l'Etat comme une bureaucratie centralisée et impersonnelle qui constituait encore l'arrière-plan théorique de la première vague de travaux sur l'histoire de l'Etat conduits dans la lignée de Theda Skocpol. Pour ce qui est du champ français, cette nouvelle approche a notamment été théorisée par Patrick Fridenson qui, s'inspirant de l'histoire des entreprises, propose de construire « une histoire de l'état contemporain comme organisation » c'est-à-dire de porter attention aux «stratégies des principaux rouages de l'Etat, l'évolution de leurs structures, en faisant toute leur place aux pratiques et aux représentations des usagers comme des agents de l'Etat » (Fridenson, 2000, lecture en ligne).

Là encore, l'histoire médiévale et moderne a ouvert la voix. Les travaux issus du programme européen sur la genèse de l'Etat ont ainsi démontré que celle-ci ne peut se comprendre que dans son rapport aux structures de pouvoir - principalement les villes et les cités - qui sont déjà là ou émergent en parallèle et avec lesquelles il est parfois en symbiose, parfois en concurrence, mais toujours en négociation et en interaction. L'étude désormais classique d'O. Raggio sur la République de Gênes aux 16 et $17^{\text {ème }}$ siècles fournit une illustration de ce type de conceptualisation (1990) ${ }^{14}$. "Dans les deux bourgs de la Fontanabuona qu'il observe, l'Etat moderne en formation est une réalité lointaine. Ce qui ne veut pas dire qu'il soit absent : mais il n'existe qu'au prix de composer avec les règles locales : celles, coutumières, de ces communautés montagnardes, et plus profondément avec celles qui sont liées au fonctionnement de la parenté » (Revel, 1995, p. 83). Plusieurs contributions de l'ouvrage déjà évoqué de J. Brewer et E. Hellmuth aboutissent à un renversement de perspective identique. En Grande-Bretagne certes mais également en Prusse, au $18^{\text {ème }}$ siècle, l'autorité et le pouvoir de contrainte de l'Etat sont d'abord le produit de la capacité de celui-ci à mettre en place une coopération avec les autorités déjà établies. Une nouvelle proposition de définition de l'Etat émerge alors : " a conglomerate of all institutions which spend public money and exert legitimate force » (Brewer et Hellmuth, 1999, p. 20). L'une des contributrices de l'ouvrage, J. Innes, suggère ainsi de porter attention à la manière dont l'Etat est le produit de ces « articulations ». Sans le savoir, elle rejoint la proposition théorique de P. Rosanvallon qui inclut également l'idée, et le terme même, d'articulation parmi les quatre dimensions auxquelles porter attention pour saisir et comprendre 1'Etat: «la déglobalisation ; la hiérarchisation ; l'articulation et la totalisation » (Rosanvallon, 1990 [1989], p. 14). Ces propositions théoriques sont particulièrement stimulantes pour la sociologie de l'Etat en ce qu'elles invitent à questionner l'équivalence entre existence d'une bureaucratie et existence de l'Etat. Ce dernier peut exercer une réelle contrainte par d'autres voies, notamment en s'appuyant sur des autorités intermédiaires et non centralisées.

Tandis qu'elle contient un dépassement de la distinction entre « centre » et " périphérie » qui a longtemps structuré les approches sociologiques classiques de l'Etat, cette proposition de renouvellement théorique trouve des échos dans plusieurs travaux récents de science politique qui ne font finalement que "redécouvrir », sans forcément y faire référence, les conclusions des historiens modernistes. L'articulation est ici saisie à l'échelle du fédéralisme. Reprenant la distinction de M. Mann entre «despotic » et «infrastructural power» (1993), D. Ziblatt compare ainsi la construction des états italiens et allemand (2006). Le force de l'Etat allemand s'explique alors par l'existence préalable de structures intermédiaires de pouvoir sur lesquelles la construction de l'Etat va s'appuyer. La conception de l'Etat comme organisation

\footnotetext{
${ }^{14}$ Pour une lecture critique par cet auteur du concept même d'Etat dans son acception courante (Raggio, 1995). Voir également (Cerutti, 1990).
} 
conduit ici à cesser de voir le fédéralisme comme un obstacle à l'action et à l'existence de l'Etat pour le considérer plutôt comme l'un des moyens possibles de son intervention, y compris coercitive. Dans le cas américain, cette perspective a conduit à reconsidérer la question du rapport entre l'Etat et les états pour ne plus les considérer dans une relation de concurrence, ou même de subsidiarité, mais pour faire de l'articulation entre ces deux composantes de l'organisation l'objet même de l'analyse (Howard, 1999) et un "pattern of American Governance » (Gerstle, 2009). K. S. Johnson montre, par exemple, comment, bien avant le New Deal et au cœur de périodes considérées comme celles du « laissez-faire », l'Etat fédéral a agi directement sur la société et l'économie tout en s'appuyant sur des acteurs nonétatiques ${ }^{15}$. Il a pour cela eu recours à ce que K. S. Johnson conceptualise sous le terme de "intergovernmental policy instruments » à l'image des prêts fédéraux aux états dont l'attribution était conditionnée à la mise en oeuvre de certaines politiques $(2007)^{16}$.

Dans le cas français, et en l'absence de fédéralisme, c'est l'Etat bureaucratique lui-même qui s'est d'abord vu décomposé comme une organisation, en un ensemble d'éléments articulés : du plus grand (le corps d'Etat et le ministère) au plus petit (le serviteur de l'Etat). L'ouverture de la boîte noire de cet Etat-organisation a ainsi donné naissance à de nombreux travaux, au croisement de l'histoire de l'Etat et de l'histoire économique : de l'étude de la direction générale des impôts (Tristram, 2005) aux directeurs généraux des douanes (Bordas, 2004 ; Clinquart, 2000) en passant par la direction du Trésor (Quennouëlle, 2000) ou encore l'organisation de la prévision économique au ministère des finances (Terray, 2002) ${ }^{17}$. A l'image de l'histoire économique, l'histoire du $19^{\text {ème }}$ siècle a de même été substantiellement transformée par cette approche (Luc, 2002 ; Stanziani, 2005 ; Karila-Cohen, 2008 ; Le Bihan, 2008 ; Galvez-Behar, 2008 et Houte, 2010). « La relation qui s'établit entre le fonctionnement intérieur de l'Etat et son action sur l'économie et la société est l'une des perspectives de l'histoire de l'Etat les plus récentes, qui devrait permettre d'apprécier les liens entre les choix délibérés des hommes politiques et des acteurs administratifs - qu'il s'agisse d'individus, de groupes, de corps ou d'institutions - et leurs effets sur les décisions publiques et donc sur la société » (Quennouëlle, 2002, p. 73).

S'ils utilisent davantage le terme de « système » que celui-ci d' $"$ organisation ${ }^{18}$, les travaux de M.-O. Baruch et V. Duclert sur l'administration, ou plus exactement sur les serviteurs de l'Etat que sont les administrateurs, relèvent également de cette approche ${ }^{19}$ (Baruch et Duclert, 2000). Ils ont ainsi porté une attention particulière aux services interministériels, d'abord sur la période $1875-1945$ puis sous la $4^{\text {eme }}$ République pour étudier moins «la nature des

\footnotetext{
${ }^{15}$ Une approche comparable se retrouve dans les travaux qui portent eux attention non à l'articulation entre Etat fédéral et états mais entre l'administration fédérale et les cours de justice locale, qui tout en étant indépendantes du pouvoir administratif, furent très tôt l'un des principaux acteurs de son implémentation à l'échelon local (Schiller, 2005).

${ }^{16}$ Pour une autre étude de cas, sur une période plus récente, Lieberman (2009).

${ }^{17}$ Pour une synthèse sur cette question voir la communication d'A. Chatriot (2010).

${ }^{18}$ Lui-même également utilisé puisqu'il est question «d'organisation de l'Etat» (Baruch et Duclert, 2003, p. 509). Les termes employés relèvent de l'organique à l'image de «machinerie de l'Etat» ou d' "appareils d'Etat» (Baruch et Duclert, 2000, p. 6-7). Dans ses premiers travaux, M.-O. Baruch emploie le terme d'ossature en précisant de quelle manière, à travers l'administration, c'est bien de l'étude de l'Etat dont il est question : « sans que l'administration se confonde juridiquement avec l'Etat, elle en constitue l'ossature » (Baruch, 1997, p. 14). Voir également Baruch et Duclert (2002).

${ }^{19}$ Ces travaux font figure d'exemple dans l'historiographie française mais ils ne sont pas les seuls à avoir réintroduit l'histoire sociale et politique de l'administration comme une voie d'accès à celle de l'Etat (Kott, 1995 ; Viet, 1994; Dard, 2002; Andrieu, 2002). Egalement pour une approche de l'administration du renseignement (Laurent, 2007 et 2004), de l'Etat en périodes de crise (Bancaud, 2002 ; Thenault, 2001) ou encore du cas soviétique (Cohen, 2003).
} 
politiques - économiques, sociales, diplomatiques, militaires etc.. - conduites par le régime que de descendre dans la salle des machines pour tenter de comprendre l'Etat [...] comme système administratif» (Baruch et Duclert (dir.), 2003, p. 505). A l'image de l'étude de l'articulation entre «Etat» et «états» au cœur du fédéralisme américain, cette approche de l'administration comme système va de pair avec l'adoption de changements d'échelles, en l'espèce hiérarchique mais aussi géographique. Fortement développée en France où elle fait figure de principale approche historienne de l'Etat, cette approche n'est pas sans parallèle Outre-Atlantique. Un des premiers historiens à se présenter comme historien de l'Etat, et rompant avec la conception tocquevillienne dominante de l'espace politique américain, B. Balogh a montré, par exemple, comment la politique énergétique nucléaire aux Etats-Unis ne pouvait se comprendre que par l'étude de l'organisation administrative alors même que la "demande sociale », tant des groupes d'intérêt économiques que des citoyens, était elle totalement inexistante (1991). Plus récemment, et venant lui de la science politique, l'ouvrage désormais classique de David Carpenter n'est pas sans écho avec l'approche de V. Duclert et M.O. Baruch puisqu'il s'intéresse également aux fonctionnaires de niveau hiérarchique moyen et à la manière dont ceux-ci se situent les uns par rapport aux autres. Par son approche des réseaux, il relève cependant plus centralement de la seconde approche, bien entendu étroitement imbriquée avec la présente. Plus récemment le développement d'une histoire transnationale de l'Etat peut être rattaché à la définition de celui-ci comme organisation. Ce n'est plus seulement sa structuration interne qui est étudiée mais la manière dont son action et sa composition s'articulent avec celles d'autres Etats ou plutôt d'autres organes dans une dynamique en réalité trans-étatique et trans-ministérielle. Les recherches de Caroline Douki, David Feldman et Paul-André Rosental sur l'articulation entre les actions des ministères du travail respectivement italien, anglais et français s'inscrivent dans cette perspective (2006).

\section{L'Etat comme relation : de la nécessité des croisements de points de vue}

Ces auteurs qualifient cependant leur démarche comme relevant d'une «histoire relationnelle ». Le terme relationnel n'est en effet pas étranger à la reconceptualisation en cours de l'Etat. Cette seconde conception recoupe pour partie la précédente. Elle s'en distingue cependant en ce qu'elle prend comme point de départ non tant les relations entre des parties de 1'Etat-organisation que celles entre Etat et Société. Là encore, cette reformulation est commune à l'ensemble des historiographies considérées. A partir de son analyse des différences entre les gestions des risques sanitaires par les Etats européens au moment des grandes épidémies, P. Baldwin suggère ainsi de dresser une typologie des Etats en fonction des modalités de leur interaction avec la société civile (2005). P. Rosanvallon considère de même que « faire l'histoire de l'Etat consiste à analyser les conditions dans lesquelles se sont formées des figures du rapport Etat/société. Car il n'y a pas d'histoire de l'Etat hors de la saisie d'une relation : l'Etat n'existe que par rapport à la société » (1990, p. 15).

Dans cette approche, il s'agit avant tout de saisir la nature "privée » des serviteurs de l'Etat comme celle "publique» des initiatives d'acteurs non-étatiques. Parce qu'elle prend l'individu comme unité d'analyse, l'histoire de l'administration construite par M.-O. Baruch et V. Duclert relève donc également de cette seconde perspective. Le « serviteur de l'Etat » y est pris en compte dans sa globalité. Il est replacé dans l'ensemble de ses relations, à travers l'étude de ses origines sociales, de sa trajectoire, de sa formation et bien sûr de ses engagements puisqu'il est question d'une « histoire politique de l'administration ». Dans cette perspective, le terme de réseaux est fortement mobilisé. Pour ce qui est de l'étude de l'administration de la $4^{\text {ème }}$ République, la conduite d'une analyse de l'Etat à la fois 
organisationnelle et relationnelle conduit ainsi ces deux auteurs à théoriser la longue absence de «réforme de l'Etat». Ils s'inscrivent ainsi en dialogue avec le renouveau de la sociologie de l'Etat porté par les travaux de P. Bezes $(2009)^{20}$. Tandis que, sous la $4^{\text {ème }}$ République, des administrations nouvelles et légères créées sur des bases relationnelles, issues des anciens réseaux de résistance et/ou de formation initiale, conduisent la réforme de la politique économique, le fonctionnement organisationnelle de l'Etat perdure sur le modèle classique de la pyramide administrative (2003). Déjà au cœur des travaux sur l'Etat moderne (Descimon et al., 1997), l'analyse de réseau est également le trait distinctif du travail de D. Carpenter qui démontre que le gain d'autonomie des administrateurs américains s'explique par leur mobilisation de nombreux réseaux au croisement des champs, notamment, économique, social et ethnique. Ici, et de manière à première vue paradoxale, c'est les relations entretenues avec le privé qui permettent aux acteurs publics de gagner en autonomie administrative et en emprise sur la société.

Sur un plan méthodologique, cette redéfinition relationnelle de l'Etat conduit à un usage extensif de prosopographie (Genet, 1997 ; Lemercier, 2003 et 2005b). La reconstitution de réseaux et de trajectoires croisées s'est ainsi largement développée ces dernières années pour devenir un point de passage obligé de l'histoire de l'Etat. Là encore, les travaux des historiens modernistes, malheureusement peu cités par les historiens du contemporain ou les politistes, ont donné lieu à d'importantes avancées conceptuelles. Au lieu de se cantonner à l'étude des assemblées représentatives, W. Reinhard a ainsi fait le choix de s'intéresser à l'ensemble des relations sociales qui existent au sein d'un groupe d'acteurs à la fois privés et publics saisis dans un continuum : ces "élites au pouvoir» (1996). Cette approche permet de saisir la genèse de l'Etat moderne non seulement à partir du haut mais également à l'aune de l'action des acteurs : du sujet du roi à l'homme du commun en passant par les membres des dites assemblées. Promu lui par des historiens spécialistes des 19 et $20^{\text {ème }}$ siècles, le projet de saisir l'Etat par ses marges a donné naissance à la notion de "pratiques consultatives de l'Etat» (Chatriot et Lemercier, 2008 et 2002) ou encore à celle d' " espace intermédiaire » (Chatriot, 2010 , p. 5) qui semblent elles aussi particulièrement opératoires. L'Etat est alors saisi à travers les relations qu'il entretient : avec le musée social (Horne, 2004), les chambres de commerce (Lemercier 2003), le conseil national économique (Chatriot, 2002), les prudhommes (Cottereau, 1987), la poste (Join-Lambert, 2001) ou encore les chambres syndicales ouvrières (Soubiran-Paillet et Pottier, 1996).

Mais c'est, sans surprise, au sein de l'historiographie américaine que cette relation entre public et privé a donné lieu à la conceptualisation la plus poussée ${ }^{21}$. Un nouveau paradigme d'analyse de l'Etat s'articule autour ce qui peut être qualifiée d' «invisibilité » de l'Etat ${ }^{22}$. Bien que l'idée soit nettement plus ancienne et que la nature hybride de l'Etat américain ait donné, depuis longtemps, lieu à des travaux historiens, sa conceptualisation comme un « hidden State » est récente. Elle est due à un politiste et est à l'origine restreinte à l'étude du Welfare State. En 1997, C. Howard a en effet proposé de redéfinir la politique sociale de l'Etat américain en ne prenant plus seulement en compte les dépenses directes mais également les déductions fiscales accordées par le gouvernement au titre de l'action sociale ("tax expenditures with social welfare purposes »). Il désigne alors cette politique sociale comme

\footnotetext{
${ }^{20}$ Notamment Baruch et Bezes (2006).

${ }^{21}$ Les débats déjà anciens qui ont suivi le développement de la micro-histoire italienne et son traitement de l'Etat semblent également porteurs de réflexions stimulantes, pour deux synthèses (Chittolini, 1995 ; Guarini, 1995). Il est à cet égard surprenant de voir que ces travaux ne sont que très rarement cités dans les débats historiens ou politistes contemporains qui semblent redécouvrir aujourd'hui certains des thèmes abordés à l'époque.

${ }^{22}$ Sur ce point précis (Gensburger, 2010).
} 
celle d'un « hidden welfare state ». Pour étudier ce dernier, il s'inscrit dans une perspective historique - ce qui en fait aujourd'hui une référence systématique des travaux historiens - en conduisant des études de cas de l'amont comme de l'aval de quatre programmes fédéraux de déductions fiscales votés respectivement en 1914, en 1926, en 1975 et 1978. Ainsi calculées, les dépenses de Welfare aux Etats-Unis atteignent un niveau comparable à celui des pays européens et ce bien avant la mise en place du New Deal. Fort de cette conclusion, C. Howard invite à rapporter les différents types d'Etat aux divers instruments de politique publique qu'ils mobilisent. Faisant finalement écho à la réflexion de K. Johnson sur l'existence d'instruments propres au fédéralisme, ce second point a, plus récemment, conduit $\mathrm{C}$. Howard à formuler un nouveau questionnement: it "may not be why the U.S. government does relatively little compared to European governments. Rather, the question is why governments of similar size devote comparable resources to pursue similar policy objectives through such a diverse mix of policy tools" (2003, p. 415). Plusieurs travaux historiens s'inscrivent dans la suite de cet intérêt pour le «hidden state ${ }^{23}$.

Un premier groupe confirme l'intuition sur la nature cachée de l'Etat américain en matière de Welfare. Il rejette cependant l'idée que les objectifs de ces politiques soient les mêmes que dans le cas des pays européens qui privilégient l'instrument de la dépense publique. Ils sont notamment beaucoup moins redistributifs et plus inégalitaires (Gottschalk, 2000 ; Klein, $2003)^{24}$. En 2002, J. Hacker a ainsi publié un ouvrage qui est désormais un classique et qui généralise l'approche de C. Howard tout en s'en distinguant sur la question de la neutralité du choix de l'instrument. Tandis que l'un prenait comme objet intermédiaire les déductions fiscales, l'autre étudie les politiques sociales assises sur l'emploi. C'est ainsi la chronologie différentielle de l'action directe de l'Etat, respectivement en matière de retraite et de sécurité sociale, qui explique la part respective qu'y jouent les canaux publics ou privés en la matière. Cependant à la différence de C. Howard, J. Hacker constate que les effets des différents types d'instruments ne sont pas le mêmes pour finalement distinguer vertical et horizontal welfare states (2006) et s'interroger : "by what standard are we to call the indirect policy tools and government-supported private benefits that are characteristic of American social provision part of that body of state activity conveniently, if often imprecisely, termed the "welfare state »? » (2005, p. 139).

Plus récemment, cette notion d' " invisibilité » de l'Etat américain a été reprise au-delà de la seule question du Welfare. M. M. Edling s'est, ainsi, intéressé à la manière dont, lors de la rédaction de la Constitution, les Fédéralistes ont développé " a conceptual framework that made it possible to accomodate the creation of a powerful national government to the strong anti-statist current in the American political tradition » (2003, p. 219). L'invisibilité de l'Etat s'est alors imposée comme la solution pour des rédacteurs qui, ayant à l'esprit les exemples européens, voulaient effectivement doter leur nouvelle nation de pouvoirs régaliens seuls susceptibles de permettre la défense du territoire et le maintien d'une indépendance récemment acquise. Ainsi, si des pouvoirs forts ont effectivement été donnés au gouvernement par la Constitution, leur exercice est d'emblée organisé pour peser non sur l'intérieur du pays et se dérouler sous les yeux des Américains mais pour s'imposer à l'extérieur et n'être visible qu'aux frontières du territoire. En lieu et place d'une conscription obligatoire qui s'imposerait aux citoyens, c'est la voie d'une armée de métier, faite d'hommes volontaires, payés et entraînés qui a été choisie tandis que les ressources fiscales devaient

\footnotetext{
${ }^{23}$ Pour une prise en compte historienne convergente du welfare (Katz, 2001 ; Jacoby, 1997).

${ }^{24}$ Sur cette question, également (Gordon, 2003).
} 
provenir du recours au protectionnisme et à la dette, principaux modes de financement d'un Etat fédéral interventionniste dès l'origine.

B. Balogh va plus loin. Il met en évidence comment, loin d'être limité à la période fondatrice, rester " out of sight» a été pour l'Etat fédéral le moyen d'agir de manière continue sur l'ensemble du $19^{\text {ème }}$ siècle pourtant considéré comme le siècle du « laissez-faire » (2009). A travers des études de cas - l'activité des cours de justice, le système postal ou encore la réglementation économique notamment -, l'auteur décrit l'organisation de son invisibilité par l'administration fédérale : l'installation des casernes militaires loin des zones de peuplement ou celle de l'administration fiscale sur les ports à distance des centres urbains. Il montre le déguisement systématique et délibéré de l'action de l'Etat sous les traits d'acteurs publics locaux. Il propose finalement une grille d'analyse qui laisse de côté l'étude des acteurs pour s'intéresser à la question de savoir comment, et à quel degré, cette nature out of sight de l'Etat américain, née d'une conjoncture particulière au moment de la rédaction de la Constitution, a pu s'institutionnaliser au cours du $19^{\text {ème }}$ siècle au point de constituer, selon l'auteur, une institution centrale de la société américaine contemporaine. On retrouve ici, sous-jacente, l'idée que le type d'instruments utilisés est susceptible de constituer la spécificité d'un mode de fonctionnement étatique. Ces divers travaux invitent enfin à s'interroger, en retour, sur la nature «visible » de l'Etat français et sur ses implications. D. King et R.C. Lieberman ont ainsi tenté de forger un modèle théorique à vocation comparative à partir de l'étude de cette redécouverte de l'Etat américain: "How American patterns offer comparative lessons 》 (2009, p. 549). Les approches qui viennent d'être détaillées font ainsi directement écho à la grille théorique multidimensionnelle proposée par ces deux auteurs. L'Etat comme organisation embrasse ainsi tant la notion d' " administrative State » que celle de "fragmented State » tandis que l'Etat comme relation correspond à celle d' « associationnal State ».

\section{L'Etat comme matrice cognitive : de la nécessité d'une histoire au second degré}

Il en va de même des deux autres dimensions mises en évidence par D.King et R.C. Lieberman. Le «standardizing State » et le « segregated State» correspondent en effet à une ultime approche historienne de l'Etat qui consiste, elle, à contourner explicitement la question de la définition de l'objet pour faire l'histoire de l'Etat «au second degré ». L'objectif est alors « de ne plus confondre les représentations idéales et en grande partie imaginaires que l'Etat, par l'intermédiaire de quelques-uns de ses théoriciens appointés, a voulu donner de luimême, et la réalité des pratiques intellectuelles, des formes de pensée et de mise en ordre du monde, des routines bureaucratiques par lesquelles il se forme, se perpétue, se légitime jour après jour avec ce que l'on appelle aujourd'hui la continuité de 1'Etat » (Bourdieu, Christin et Will, 2002, p. 6). L'idée sous-jacente est que l'Etat «se perpétue par les catégories par lesquelles il pense et donne à penser ce qu'il pense » (Lenoir, 2000, p. 96). En un mot, il est « une forme efficace de représentation sociale » (Rosanvallon, 1990, p. 14).

En lien avec les approches de l'Etat comme organisation et comme relation, l'étude, conduite par P. Minard, du colbertisme, à partir de celle du corps des inspecteurs des manufactures, relève ainsi de cette appréhension de l'Etat comme matrice cognitive (1998). Cette histoire concrète des pratiques administratives de contrôle de la société donne en effet à voir la manière dont l'Etat encadre la vie économique et la normalise, au sens fort du terme. En s'intéressant à la manière dont les fabricants ont réagi à l'institutionnalisation de contrôle étatique, P. Minard parvient notamment à saisir cette imbrication entre Etat et société. Un certain nombre de travaux sur la statistique s'inscrit également dans cette perspective. En considérant que les représentations sont des réalités et en produisent, l'histoire des catégories 
et du calcul statistiques constitue ainsi un domaine privilégié d'étude de l'Etat (Desrosières, 2008, 2005 et 1993 ; Karady, 2008). Aux Etats-Unis, W. R. Childs a mis en évidence comment, sur la période 1889 à 1945, l'Etat central américain a utilisé les statistiques pour uniformiser la politique de régulation qu'il mettait en place de manière pourtant indirecte puisque à travers les états (Childs, 2001; Haggerty, 2002). Plus largement l'étude des questions raciales aux Etats-Unis a donné lieu à de nombreux travaux sur les catégories démographiques, à l'image des travaux de D. King (1995). En France, l'ouvrage d'E. Brian sur la «mesure de l'Etat» est lui exemplaire. L'auteur y retrace l'histoire administrative et intellectuelle des dénombrements de population dans la France du $18^{\text {ème }}$ siècle, en prenant l'œuvre de Colbert comme fil rouge (1994). Cet ouvrage fait notamment le lien entre la division du travail statistique et la nature de ce travail saisie à travers la catégorisation du monde qu'il produit. L'Etat est alors saisi au-delà de la distinction entre représentations et réalités. L'ouvrage contemporain, et désormais classique, d'Alain Desrosières suggère lui que l'histoire et les contours différentiels de la statistique administrative permettent de comparer les conceptions et l'organisation des Etats entre eux - en l'espèce pour les cas français, britannique, allemand et américain. Comme, à partir de l'étude du cas soviétique, Alain Blum et Catherine Gousseff le résument clairement, l'histoire de la statistique démographique est un " domaine qui revendique une forte perspective comparative et qui s'offre comme un point de vue privilégié pour appréhender certains aspects de la construction de l'Etat, des relations entre administration et pouvoir politique d'une part, et entre Etat et société, de l'autre, à travers notamment la construction des représentations sociales » (1997, p. 441). Ces recherches, menées à l'origine de manière relativement isolées les unes des autres, ont finalement dessiné ce que P.-A. Rosental a qualifié d'《 histoire politique des populations » (2006 et 2003). Celle-ci tente de saisir les processus d'«auto-création » des sociétés et, notamment d'y cerner le rôle d'un Etat conçu d'emblée comme organisation et relation, à l'image des travaux de P.-A. Rosental sur l'interministériel en matière de travail ou encore de migration (2009).

Poussée à sa limite, cette approche appelle une réflexion épistémologique sur la manière dont l'histoire comme discipline a conceptualisé l'Etat, à travers le temps et l'espace. La focale se situe alors au croisement de l'histoire sociale et de l'histoire des idées. Le livre classique de Q. Skinner constitue à cet égard un modèle (1978). Pour le cas français, l'ouvrage de P. Rosanvallon sur les limites d'une approche en termes de jacobinisme consacre une large place à l'analyse du succès qu'a, jusqu'à récemment, rencontré ce concept pour penser l'Etat français (2004). L'article de W. Novak sur le mythe de la faiblesse de l'Etat américain s'engage sur cette voie (2008) tout comme le texte de D. King et M. Stears (2009). Ces derniers s'y interrogent sur les raisons du peu d'intérêt de la science politique américaine pour l'Etat après 1945. Cette troisième composante des travaux historiens sur l'Etat nous apprend ainsi que toute réflexion sur les recompositions contemporaines de celui-ci devrait inclure une réflexion au second degré sur les recompositions du regard que le chercheur, historien mais aussi sociologue, pose sur cet objet.

\section{Références bibliographiques}

Andrieu C., 2002. - Pour l'amour de la République: le club Jean-Moulin : 1958-1970, Paris, Fayard.

Badie B., Birnbaum P., 1979. - La Sociologie de l'Etat, Armand Colin, Paris.

Baldwin P., 2005. - "Beyond Weak and Strong : Rethinking the State in Comparative Policy History", Journal of Policy History, 17, 1, pp. 12-33. 
"Contributions historiennes au renouveau de la sociologie de l'Etat. Regards croisés franco-américains ", Revue française de sociologie, rubrique Note critique, printemps 2011, p. 579-602.

Baldwin P., 2003. - "The Return of the Coercive State? Behavioral Control in Multicultural Society" dans J. A. Hall et al. (dir.), The Nation-State Under Challenge:Autonomy and Capacity in a Changing World, Princeton University Press, pp. 106-136.

Baldwin P., 1999. - Contagion and the State in Europe, 1830-1930, Cambridge University Press.

Balogh B., 2009. - A Government out of sight. The Mystery of National authority in nineteenth-century America, Cambridge University Press.

Balogh B., 2003. - "The State of the State among Historians », Social Science History, 27, 3, pp. 455-463.

Balogh B., 1991. - Chain Reaction : Expert Debate and Public Participation in American Commercial Nuclear Power, 1945-1975, NY, Cambridge University Press.

Bancaud A., 2002. - Une exception ordinaire : la magistrature en France, 1930-1950, Paris, Gallimard.

Barringer Gordon S., 2002. - The Mormon Question : Polygamy and Constitutional Conflict in Nineteenth-Century America, Chapel Hill, N.C., University of North Carolina Press.

Baruch M.-O., Bezes P. (dir.), 2006. - «Généalogie de la réforme de l'Etat», Revue française de l'administration publique, 120.

Baruch M.-O., Duclert V., 2003. - «Administrateurs et administrations sous la IVe République : une histoire politique », Revue française d'administration publique, 4, 108, pp. 501-519.

Baruch M.-O., Duclert V., 2002. - Justice, politique et République. De l'Affaire Dreyfus à la guerre d'Algérie, Bruxelles, Complexe / IHTP-CNRS.

Baruch M.-O., Duclert V., 2000. - Serviteurs de l'Etat. Une histoire politique de l'administration française. 1875-1945, Paris, La Découverte.

Baruch M.-O., 1997. - Servir l'Etat français. L'administration en France de 1940 à 1944, Paris, Fayard.

Bensel R.F., 2000. - The Political Economy of American Industrialization, 1877-1900, NY, Cambridge University Press.

Bensel R.F., 1991. - Yankee Leviathan : The Origins of Central State Authority in America 1859-1877, NY, Cambridge University Press.

Bezes P., 2009. - Réinventer l'Etat. Les réformes de l'administration française (1962-2008), Paris, PUF.

Blum A., Gousseff G., 1997. - « La statistique démographique et sociale, élément pour une analyse historique de l'Etat russe et soviétique », Cahiers du monde russe, 38, 4, pp. 441-455.

Bordas J., 2004. - Les directeurs généraux des douanes : l'administration et la politique douanière, 1801-1939, MEIF, Comité d'histoire.

Boucheron P., 1998. - «Ecrire autrement l'histoire politique », Médiévales, printemps, pp. 711.

Bourdieu P., Christin O., Will P.E., 2002. - «Sur la science de 1'Etat», Actes de la Recherche en Sciences Sociales, 133, 1, pp. 3-11.

Braddick M.J., 1996. - The Nerves of State: Taxation and the Financing of the English State, 1558-1700, Manchester University Press.

Brian E., 1994. - La Mesure de l'Etat. Administrateurs et géomètres au XVIIIe siècle, Paris, Albin Michel.

Brewer J., Hellmuth, E. (dir.), 1999. - Rethinking Leviathan : The Eighteenth-Century State in Britain and Germany, NY, Oxford University Press.

Capuano C., 2009. - Vichy et la famille. Réalités et faux-semblants d'une politique publique, Rennes, Presses Universitaires de Rennes, préface de Paul-André Rosental. 
"Contributions historiennes au renouveau de la sociologie de l'Etat. Regards croisés franco-américains ", Revue française de sociologie, rubrique Note critique, printemps 2011, p. 579-602.

Carpenter D.P., 2001. - The Forging of Bureaucratic Autonomy : Reputations, Networks, and Policy Innovation in Executive Agencies, 1862-1828, Princeton, Princeton University Press.

Cerutti S., 1990. - La Ville et les métiers. Naissance d'un langage corporatif (Turin, XVIIXVIII siècles) Paris, Editions de l'EHESS.

Charle C., 1987. - Les élites de la République, 1880-1900, Paris, Fayard.

Chatriot A., Lemercier C., 2008. - «Une histoire des pratiques consultatives de 1'État», dans Michel Offerlé et Henry Rousso (dir.), La fabrique interdisciplinaire. Histoire et science politique, Rennes, Presses universitaires de Rennes, pp. 191-203.

- 2002. - «Les corps intermédiaires » dans Vincent Duclert et Christophe Prochasson (dir.), Dictionnaire critique de la République, Paris, Flammarion, pp. 691-698.

Chatriot A., 2006. - "L'Etat, un objet paradoxalement neuf pour l'histoire politique contemporaine française » dans Alain Chatriot et Dieter Gosewinkel (dir.), Figurationen des Staates in Deutschland und Frankreich 1870-1945. Les figures de l'Etat en Allemagne et en France, Münich, Oldenbourg Verlag, pp. 7-17.

- 2010. - «L'évidence de l'Etat, une difficulté, chronique pour les historiens politiques français », Colloque « Sortir du paradigme de l'action », Sciences Po, 10-12 mai, pp. 1-8.

- 2002. - La démocratie sociale à la française. L'expérience du Conseil national économique, 1924-1940, Paris, La Découverte.

Childs W.R., 2001. - « State Regulators and Pragmatic Federalism in the United States, 1889-1945 », Business History Review, 75, Hiver, pp. 701-738.

Chittolini G., 1995. - « the 'Private', the 'Public', the State », Journal of Modern History, 67, décembre, p. S34-S61.

Clinquart J., 2000. - L'Administration des douanes en France de 1914 à 1940, Paris, MEIF, Comité d'histoire.

Cohen Y., 2003. - « Administration, politique et techniques. Réflexions sur la matérialité des pratiques administratives dans la Russie stalinienne (1922-1940) », Cahiers du Monde russe, 44, 2-3, pp. 269-308.

Cottereau A. (dir.), 1987. - «Les prud'hommes XIX ${ }^{\mathrm{e}}-\mathrm{XX}^{\mathrm{e}}$ siècle », Le Mouvement social, 141.

Dard, O., 2002. - Le rendez-vous manqué des relèves des années trente, Paris, PUF.

Daunton M., 2001. - Trusting Leviathan: the politics of taxation in Britain, 1799-1914, NY, Cambridge University Press.

- 2002. - Just Taxes: the politics of taxation in Britain, 1914-1979, Cambridge University Press.

Delalande N., 2009. - «Le consentement de l'impôt en France: les contribuables, l'administration et le problème de la confiance. Une étude de cas en Seine-et-Oise (années 1860-années 1930) », Revue d'Histoire Moderne et Contemporaine, 56-2, pp. 135-163.

Descimon R., Guéry A., 1989. - "Un Etat des temps modernes ?", dans A. Burguière et J. Revel (dir), Histoire de la France, vol 2, L'Etat et les pouvoirs, Paris, Seuil, pp. 181-356.

Descimon R., Schaub J.F., Vincent B. (dir.), 1997. - Les figures de l'administrateur : institutions, réseaux, pouvoirs en Espagne, en France et au Portugal, $16^{e}-19^{e}$ siècle, Paris, EHESS.

Desrosières A., 2008. - L'argument statistique (tome I et II), Paris, Mines Paris Tech - Les Presses.

- 2005. - "Décrire 1'Etat ou explorer la société : les deux sources de la statistique publique", Genèses, 58, pp. 4-27.

- 1993. - La politique des grands nombres - histoire de la raison statistique, Paris, La Découverte. 
"Contributions historiennes au renouveau de la sociologie de l'Etat. Regards croisés franco-américains ", Revue française de sociologie, rubrique Note critique, printemps 2011, p. 579-602.

Douki C., Feldman D., Rosental P.A., 2006. - « Pour une histoire relationnelle du ministère du Travail en France, en Italie et au Royaume-Uni dans l'entre-deux-guerres : le transnational, le bilatéral et l'interministériel en matière de politique migratoire » dans A. Chatriot, O. Join Lambert et V. Viet (dir.), Les politiques du Travail (1906-2006), Presses Universitaires de Rennes, pp. 143-159.

Dutton P., 2007. - Differential Diagnoses. A Comparative History of Health Care Problems and Solutions in the United States and France, Ithaca, Cornell University Press.

- 2002. - Origins of the French Welfare State: The Struggle for Social Reform in France 1914-1947, New York, Cambridge University Press.

Dyson K., 1980. - The State Tradition in Western Europe. A Study of an Idea and Institution, Oxford, Martin Robertson.

Edling M.M., 2003. - A Revolution in Favor of Government: Origins of the U.S. Constitution and the Making of the American State, NY, Oxford University Press.

Ertman T., 1997. - The birth of the leviathan: Building states and regimes in medieval and early modern Europe, Cambridge, Cambridge University Press.

Fehrenbacher D.E., 2001. - The Slaveholding Republic : An Account of the United States Government's Relations to Slavery, NY, Oxford University Press.

Fridenson P., 2000. - «Pour une histoire de l'état contemporain comme organisation », Les Cahiers du Centre de Recherches Historiques, 25, mis en ligne le 16 janvier 2009.

Galvez-Behar G., 2008. - La République des inventeurs. Propriété et organisation de l'innovation en France (1791-1922), Rennes, Presses universitaires de Rennes.

Genet J.-P., 1997. - «La Genèse de l'Etat Moderne. Les enjeux d'un programme de recherche », Actes de la Recherche en Sciences Sociales, 118, 1, pp.3-18.

Gensburger S., 2010. - «La main invisible de l'Etat Américain», Revue française de science politique, 5, pp. 1023-1030

Gerstle G., 2009. - « The Resilient Power of the States across the Long Nineteenth Century. An Inquiry into a Patern of American Governance", dans L. Jacobs et D. King, 2009, pp. 6187.

Gordon C., 2003. - Dead on Arrival : the Politics of Health Care in Twentieth-Century America, Princeton, Princeton University Press.

Gordon L., 1994. - Pitied but not entitled: Single Mothers adn the History of Welfare 18901935, Cambridge, Harvard Univeristy Press.

Gorski P.S., 2001. - "Beyond Marx and Hintze ? Third-Wave Theories of Early Modern State Formation", Comparative Studies in Society and History, 43, 4, pp. 851-861.

Gosewinkel D., 2006. - « Histoire et historiographie de 1'Etat en France et en Allemagne », dans Alain Chatriot et Dieter Gosewinkel (dir.), Figurationen des Staates in Deutschland und Frankreich 1870-1945. Les figures de l'Etat en Allemagne et en France, Münich, Oldenbourg Verlag, pp. 19-30.

Gottschalk M., 2000. - The Shadow Welfare State. Labor, Business, and the Politics of Health Care in the United States, Ithaca, Cornell University Press.

Graham H.D., 1993. - « The Stunted Career of Policy History : A critique and an Agenda », The Public Historian, 15, pp. 15-37.

Gribaudi M., 2009. - « Le savoir des relations : liens et racines sociales d'une administration dans la France du XIX siècle », Le Mouvement social, 3, 228, pp.9-38.

- 1998. - «Des micro-mécanismes aux configurations globales : causalité et temporalité historiques dans les formes d'évolution de l'administration française au XIXème siècle » in J. Schlumbohm (dir.), Mikrogeschichte Makrogeschichte - Komplementär oder inkommensurabel ?, Göttingen, Wallstein Verlag, pp. 83-128. 
"Contributions historiennes au renouveau de la sociologie de l'Etat. Regards croisés franco-américains ", Revue française de sociologie, rubrique Note critique, printemps 2011, p. 579-602.

Gribaudi M., Magaud J. (dir.), 1999. - L'action publique dans les domaines sanitaire et social en France de 1800 à 1900, convention MIRE-INED.

Fasano Guarini E., 1995. - «Center and Periphery », Journal of Modern History, 67, p. S74-S96.

Guery A., 1997. - «L'historien, la crise et l'État», Annales. Histoire, Sciences Sociales, 52, 2, pp. $233-256$.

Hacker J.S., 2006. - The Great Risk Shift : The Assault on American Jobs, Families, Health Care, and Retirement and How You Can Fight Back, NY, Oxford University Press.

- 2005. - "Bringing the Welfare State Back In: The Promise (and Perils) of the New Social Welfare History », Journal of Policy History, 17, 1, pp.125-154.

- 2002. - The Divided Welfare State : The Battle over Public and Private Social Benefits in the United States, NY, Cambridge University Press.

Hays S.P., 1987. - Beauty, Health and Permanence. Environmental Politics in the United States 1955-1985, NY, Cambridge University Press.

Horne J., 2004. - Le Musée social. Aux origines de l'Etat providence, Pairs, Belin.

Houte A.-D., 2010. - Le métier de gendarme au XIXe siècle, Rennes, PUR.

Howard C., 2003. - "Is the American Welfare State Unusually Small ?", PS : Political Science and Politics, 36, 3, pp. 411-416.

- 1999. - “The American Welfare States, or States ?”, Political research Quarterly, 52, 2, pp. 421-442.

- 1997. - The Hidden Welfare State : Tax Expenditures and Social Policy in the United States, Princeton, NJ, Princeton University Press.

Jacobs L., King D., 2009. - The Unsustainable American State, New York, Oxford University Press.

Jacoby S.M., 1997. - Modern Manors : Welfare Capitalism since the New Deal, Princeton, Princeton University Press.

John R.R. (dir.), 2006. Ruling Passions : Political Economy in Nineteenth Century America, University Park, Pa.

- 1995. - Spreading the News: the American Postal System from Franklin to Morse, Cambridge, Harvard University Press.

Johnson K.S., 2007. - Governing the American State : Congress and the New Federalsim, 1877-1929, Princeton, Princeton University Press.

Join-Lambert O., 2001. - Le receveur des Postes, entre l'Etat et l'usager 1944-1973, Paris, Belin.

Karady V., 2008. - «Les fonctions idéologiques des statistiques confessionnelles et ethniques dans la Hongrie post-féodale (1867-1948)», Revue d'histoire des sciences humaines, 1, 18, pp. 17-34.

Karila-Cohen P., 2008. - L'État des esprits. L'invention de l'enquête politique en France (1814-1848). Rennes, PUR.

Katz M.B., 2001. - The Price of Citizenship : Redefining the American Welfare State, NY, Metropolitan Books.

King D., Lieberman R.C., 2009. - "Ironies of State building. A Comparative Perspective on the American State", World Politics, 3, pp. 547-588.

King D., Stears M., 2009. - « The Missing State in Postwar American Political Thought" dans Lawrence Jacobs and Desmond King (eds.), The Unsustainable American State, New York, Oxford University Press.

King D., 1995. - Separate and Unequal: African Americans and the US Federal Government, Oxford University Press. 
"Contributions historiennes au renouveau de la sociologie de l'Etat. Regards croisés franco-américains ", Revue française de sociologie, rubrique Note critique, printemps 2011, p. 579-602.

Klein J., 2003. - For All These Rights : Business, Labor, and the Shaping of America's Public-Private Welfare State, Princeton, NJ, Princeton University Press.

Kott S., 1995. - L'Etat social allemand : représentations et pratiques, Paris, Belin.

Laborde C., 2000. - Plualist thought and the state in Britain and France, 1900-1925, Londres, Macmillan.

- 2000. - "Penser l'Etat en Grande-Bretagtne" dans Marc-Olivier Baruch et Vincent Duclert, Serviteurs de l'Etat. Une histoire politique de l'administration française. 1875-1945, Paris, La Découverte, pp. 68-82.

Laurent S., 2007. - "La naissance du renseignement étatique en France au XIX siècle entre bureaucratie et politique", Revue d'histoire du XIX, 35, 2, pp. 109-124.

- 2004. - « Pour une autre histoire de l'Etat», Vingtième siècle, 83, 3, pp. 173-184.

Lavau G., 1980. - «A propos de trois livres sur l'Etat», Revue française de science politique, 30, 2, pp. 396-412.

Le Bihan J., 2008. - Au service de l'Etat. Les fonctionnaires intermédiaires au XIXe siècle, rennes, PUR.

Lemercier C., 2005a. - « La France contemporaine : une impossible société civile ? », Revue d'Histoire Moderne et Contemporaine, 52-3, pp. 166-179.

- 2005b. - « Analyse de réseaux et histoire », Revue d'histoire moderne et contemporaine, 522, p. 88-112.

- 2003. - Un si discret pouvoir. Aux origines de la chambre de commerce et d'industrie de Paris 1803-1853, Paris, La Découverte.

Lenoir R., 2000. - «Savoirs et sciences d'état: généalogie et démographie », Actes de la recherche en sciences sociales, 133, 1, pp.96-97.

Leuchtenburg W., 1986. "The pertinence of political history: Reflections on the significance of the state in America », Journal of American History, 73, pp. 585-600.

Lieberman R., 2009. - "Civil Rights and the Democratization Trap : The Public-Private Nexus and the Building of American Democracy », dans D. King et alii., Democratization in America. A Comparative-Historical Anlysis, Baltimore, John Hopkins University, pp. 211229

Luc J.-N. (dir.), 2002. - Gendarmerie, Etat et société au XIXe siècle, Paris, Publications de la Sorbonne.

Mann M., 1993. - The Sources of Social Power, NY, Cambridge University Press.

- 1986. - "The autonomous Power of the State : Its Origins, Mechanisms and Results" dans John A. Hall (dir.), States in History, NY, Oxford University Press, pp. 109-136.

Mann P., 2000. - «La genèse de 1'Etat moderne : Max Weber revisité », Revue française de sociologie, 41-2, p p. 331-344.

Margairaz M., 1991. - L'Etat, les Finances et l'Economie : histoire d'une conversion 19321952, Paris, MEIF, Comité d'histoire.

Mashaw J.L., 2005. - « Recovering American Administrative Law : Federalist Foundations, 1787-1801 », Yale Law Journal, 115, pp. 1258-1244.

Meadowcroft J., 1995. - Conceptualizing the State. Innovation and Dispute in British Political Thought 1880-1914, Oxford, Clarendon Press.

Minard P., 1998. - La Fortune du colbertisme. Etat et industrie dans la France des Lumières, Paris, Fayard.

Nettl J.P., 1968. - "The State as a Conceptual Variable", World Politics, 4, pp. 559-592.

Ngai M.N., 2005. - Impossible Subjects : Illegal Aliens and the Making of Modern America, Princeton, N.J., Princeton University Press.

Novak W.J., 2008. - "The Myth of the "Weak» American State", American Historical Review, pp. 752-77. 
"Contributions historiennes au renouveau de la sociologie de l'Etat. Regards croisés franco-américains ", Revue française de sociologie, rubrique Note critique, printemps 2011, p. 579-602.

- 1996. - The People's Welfare : Law and regulation in Nineteenth-Century America, Chapel Hill, University of North Carolina Press.

Pierson P., 2005. - « The Study of Policy Development», Journal of Policy History, 17, 1, pp.34-51.

Quennouëlle L., 2002. - « Où va l'histoire de 1'Etat? », Le Mouvement social, n²00, pp. 7379.

- 2000. - La Direction du Trésor, 1947-1967: L'Etat-banquier et la croissance, MEIF, Comité d'histoire.

Raggio O., 1995. - «Visto dalla perfieria. Formazione politiche di anticho regime e Stato moderno » dans Maurice Aymard, (dir.), Storia d'Europa. L'età moderna : secoli XVI-XVIII, Turin, Einaudi, pp. 483-527.

- 1990. - Faide e parentele. La stato genovese visto dalla Fontanabuona, Turin.

Reinhard W. (dir.), 1996. - Les élites du pouvoir et la construction de l'Etat en Europe, Paris, PUF.

Revel J., 1995. - «L'institution et le social» dans Bernard Lepetit (dir.), Les formes de l'expérience. Une autre histoire sociale, Paris, Albin Michel, pp. 63-84.

Rosanvallon P., 1990. - L'Etat en France de 1789 à nos jours, Paris, Seuil.

- 2004. - Le modèle politique français. La société civile contre le jacobinisme de 1789 à nos jours, Paris, Seuil.

Rosental P.A., 2009. - «Health and Safety at Work: An issue in Transnational History. Introduction", Journal of Modern European History, 7, 2.

- 2006. - « Pour une histoire politique des populations », Annales HSS, 1, pp. 7-29.

- 2003. - L'intelligence démographique. Sciences et politiques des populations en France (1930-1960), Paris, Odile Jacob.

Schaub J.-F., 1996. - «Le temps et l'Etat: vers un nouveau régime historiographique de l'Ancien Régime français », Quaderni Fiorentini, 25, pp. 127-181.

Schiller R., 2005. " «Saint George and the Dragon »: Courts and the Development of the Administrative State in Twentieth-Century America », Journal of Policy History, 17, 1, 2005, pp. 110-124.

Skinner Q., 1978. - The Foundations of modern political thought, NY, Cambridge University Press.

Skowronek S., 1982. - Building a New American State. The Expansion of National Administrative Capacities, 1877-1920, Cambridge.

Soubiran-Paillet F., Potier M.L., 1996. - De l'usage professionnel à la loi : les chambres syndicales ouvrirères parisiennes de 1867 à 1884, Paris, L'Harmattan, 1996.

Stanziani A., 2005. - Histoire de la qualité alimentaire XIXe-XXe siècle, Paris, Le Seuil.

Stasavage D., 2003. - Public Debt and the Birth of the Democratic State : France and Great Britain, 1688-1789, NY, Cambridge University Press.

Stearns P.N., 1999. - Battleground of Desire : The Struggle for Self-Control in Modern America, NY, New York University Press.

Te Brake W., 1998. - Shaping History. Ordinary People in European Politicis, 1500-1700, Berkeley, University of California Press.

Terray A., 2002. - Des francs-tireurs aux experts: l'organisation de la prévision économique au ministère des finances, 1948-1968, MIEF, Comité d'histoire.

Thénault S., 2001. - Une drôle de justice : les magistrats dans la guerre d'Algérie, Paris, La Découverte.

Tilly C., 1990. - Coercion, Capital and European States, AD 990-1990, Cambridge, B. Blackwell. 
"Contributions historiennes au renouveau de la sociologie de l'Etat. Regards croisés franco-américains ", Revue française de sociologie, rubrique Note critique, printemps 2011, p. 579-602.

Tilly C., 1975. - The Formation of National States in Western Europe, Princeton, Princeton University Press.

Tomlins C., 2008. - «Necessities of State: Police, Sovereignty and the Constitution», Journal of Policy History, 20, 1, pp.47-63.

Tristram F., 2005. - Une fiscalité pour la croissance : la direction générale des impôts et la politique fiscale en France de 1948 à la fin des années 1960, Paris, MEIF, Comité d'histoire.

Viet V., 1994. - Les voltigeurs de la République. L'inspection du travail en France jusqu'en 1914, 2 vol., Paris.

White R., 1991. - "It's Your Misfortune and None of my Own» : A New History of the American West, Norman, University Oklahoma Press.

Wilson M.R., 2006. - The Business of Civil War : Military Mobilization and the State, 18611865, Baltimore, Md., John Hopkins University Press.

Zelizer J.E., 2005. - "Introduction : New Directions in Policy History", Journal of Policy History, 17, 1, pp. 1-11.

- 2004. - "History and Political Science : together again ?", Journal of Policy History, 16,2, pp. 126-136.

- 2003. - "Stephen Skowronek's Building a New American State and the Origins of American Political Develpoment", Social Science History, 27, 3, pp. 425-441.

- 2000. - "Clio's Lost Tribe : Public Policy History since 1978”, Journal of Policy History, 12, 3, pp. 369-394.

Ziblatt D., 2006. - Structuring the State: The Formation of Italy and Germany and the Puzzle of Federalism, Princeton, Princeton University Press. 